\section{Bulking up immunity with instant Abs}

\section{By Michael J. Haas, Senior Writer}

When Pfizer Inc. bought CovX Pharmaceuticals Inc. in 2007, the driver of the deal was the biotech's CovX-Body technology, which used a single antibody as a scaffold on which to build multiple product candidates that could be given via infusion. Pfizer may earn bonus miles from its acquisition, as CovX's cofounder at The Scripps Research Institute has now devised a way to produce the antibody therapeutics entirely in vivo, potentially obviating the need for infusions.

The technology, which is already licensed to Pfizer, uses a vaccine to induce the antibody scaffold, then a chemical agent to modify and direct the antibody against a specific disease target, thereby providing what the Scripps team has termed "instant immunity."

The group has shown proof of principle in mouse models of cancer and thinks the technology for chemically programmed antibodies could work against infectious diseases.

Unlike most antibodies, which bind their targets noncovalently, the antibody scaffolds used by Pfizer and Scripps are capable of binding their targets covalently, so that antibody and target fuse into a single molecule. Such antibodies were first reported in 1995 by a team led by Carlos Barbas III, chair of molecular biology at Scripps. The group found that mice injected with a small molecule (1,3-diketone) always developed antibodies that could covalently bind that molecule. ${ }^{1}$

According to team member Richard Lerner, now president at Scripps as well as a professor of chemistry and immunochemistry, this led to the hypothesis that the antibodies could be programmed against disease targets by linking a target-specific molecule to the antibodyinducing diketone.

Lerner and Barbas formed CovX in 2002 to commercialize these antibodies.

\section{Abs training}

The first-generation therapeutics are produced ex vivo and must be delivered by infusion. The latest Scripps work, also led by Barbas, investigated whether therapeutic antibodies could be produced and programmed entirely in vivo.

First, the team ascertained whether the anti-diketone antibody scaffold could find and bind a programming agent in vivo. To do this, they inoculated healthy mice with a vaccine based on a 1,3-diketone. Sixty-five days later they injected the mice with a programming agent composed of a diketone linked to an inhibitor of two antiangiogenic integrins: integrin $\alpha_{\mathrm{v}} \beta_{3}$ and integrin $\alpha_{\mathrm{v}} \beta_{5}$.
Experiments on serum pooled from the treated mice confirmed that the antibodies and programming agent had indeed found one another in vivo and fused into programmed antibodies (see Figure 1, "Production of chemically programmed antibodies in vivo").

The team repeated the treatment with the vaccine and programming agent in mouse models of colon cancer and melanoma and observed decreases in tumor growth of more than $75 \%$ compared with that seen in controls.

The findings were published in the Proceedings of the National Academy of Sciences. ${ }^{2}$

In the paper, the team also suggested that programmed antibodies could induce immunity against infectious diseases such as HIV, malaria and pandemic flu.

To do so, Lerner told SciBX that humans could be inoculated with the diketone at any time to induce the programmable antibodies. "You could then give a chemical programming agent as needed to activate this reservoir of antibodies" against an emerging disease or even a chemical threat such as nerve gas to confer immunity almost instantaneously, he said. Lerner added that chemical programming agents could be taken orally.

Barbas noted that the approach would have lower production and prescription costs than infused antibody therapies.

\section{Abs vantage points}

Other researchers agreed that the technology is ready to test in cancer and potentially autoimmune disease, but they said direct evidence for its effectiveness against infectious disease is lacking.

Fons Uytdehaag, senior director of R\&D strategy development at vaccine company Crucell N.V., said the Scripps approach has a big advantage over immunization with recombinant or vectored vaccines because it induces a universal immune response and memory, then rapidly directs that response against a disease target with the chemical programming agent.

Eric Guenzi, associate director of immunology research at cancer and autoimmune disease company MediGene AG, agreed that the instant immunity conferred by programmed antibodies offers a major selling point over conventional vaccines or infused antibody therapy.

"You could use this approach to re-create and reprogram therapeutic agents-which previously would have to be injected-by inducing instant immunity through vaccination with reactive compounds," he said. "With this system, your body does the job."

Guenzi said the results in cancer were promising, but he wanted to see animal studies showing that programmed antibodies could induce regression in established tumors and prevent metastasis. "It would be highly relevant to check the efficacy of the reprogrammed antibodies after the tumor has been established" for 2-3 weeks instead of beginning treatment before increasing tumor volume or tumor growth were measurable, as the Barbas collaborators did in their study, he said.

Guenzi also wanted to see experiments against more established cancer targets. "To my knowledge, the efficacy of inhibitors of $\alpha_{\mathrm{v}} \beta_{3}$ and $\alpha_{\mathrm{v}} \beta_{5}$ integrins has not been established in the clinic," he said. 


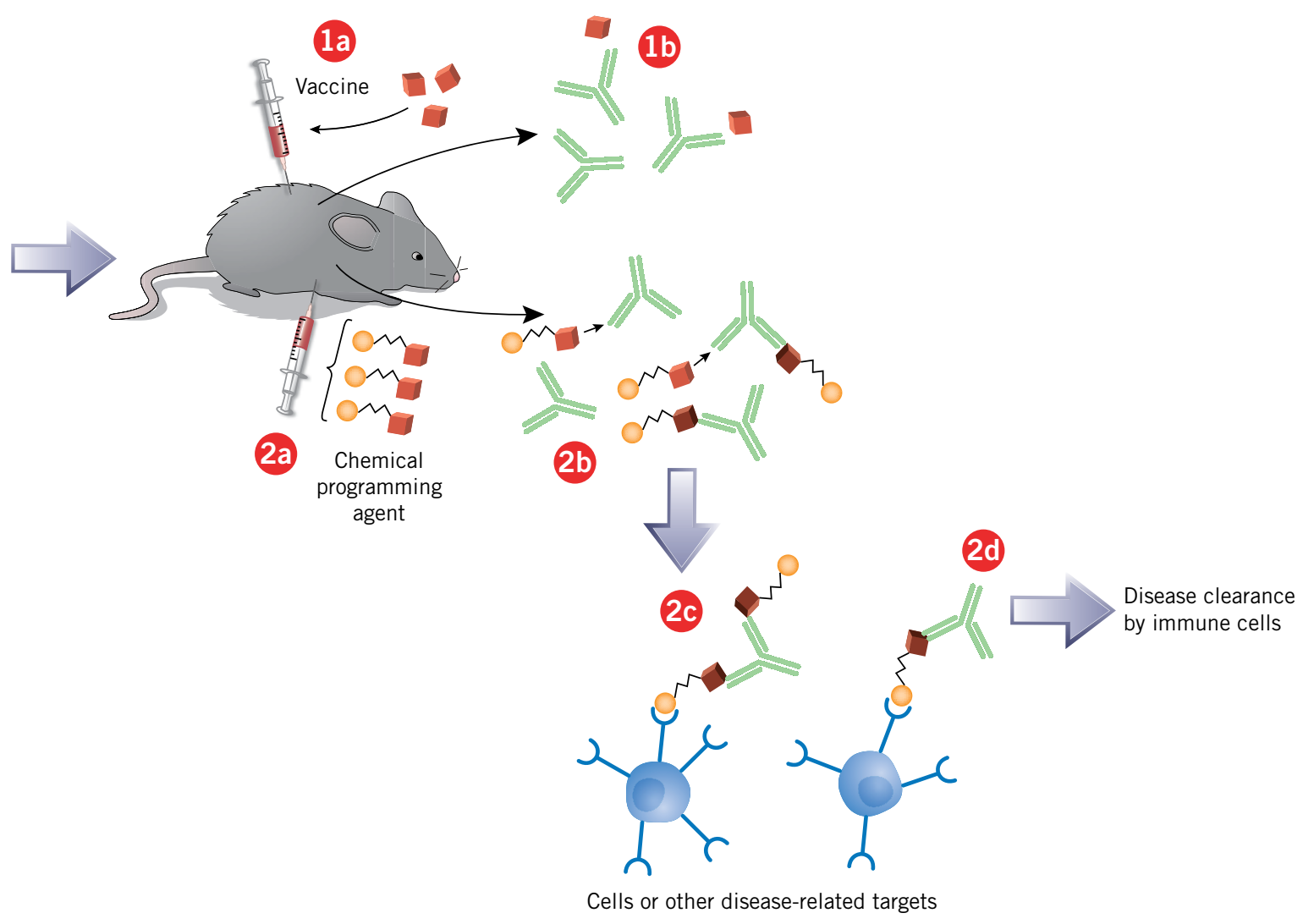

Figure 1. Production of chemically programmed antibodies in vivo. In a paper in the Proceedings of the National Academy of Sciences, Popkov et al. showed a process for generating chemically programmed antibodies in mice:

[1a] In the first stage of the process, mice are inoculated with a vaccine consisting of a specific kind of small molecule, a 1,3-diketone (brown cube).

[1b] As a result, mice generate an antibody response against the diketone molecule.

[2a] In the second stage, the immunized mice are injected with a chemical programming agent composed of the diketone linked to a therapeutic moiety (yellow circle), designed to bind a disease target.

[2b] The diketone moiety of the programming agent re-elicits the antibody response triggered in the first stage and covalently binds to the anti-diketone moiety of the generated antibodies.

[2c] The antibodies are now programmed to bind to the disease target via the therapeutic moiety.

[2d] The target-programmed antibody complex is cleared by the immune system following binding to the programmed antibody's Fc region.

Nevertheless, Guenzi noted that past approaches to treating cancer with therapeutic antibodies required adjuvant therapy. "Here they showed inhibition of tumor growth without recourse to adjuvant therapy, which is a clear advantage," he said.

Guenzi said the Barbas team's approach also allowed control over the immune response to self-antigens, which would avoid long-term safety issues that could arise when vaccinating against self-antigens.

"This is the first time I've read about something that combines an immunization approach with a short, intense reaction that controls the immune response and the memory of immune response," he said. "If you stop taking the programming agent, you stop the reactions, making the immune response controllable and reversible. This is a very important point for both cancer and autoimmune conditions."

Robert Rickert, associate professor of inflammatory diseases at the Burnham Institute for Medical Research, agreed that the technology provides a broad platform that could apply to multiple diseases. "The key will be to find appropriate therapeutic molecules or peptides that can be combined with the diketone" to make programming agents that are as effective as those used in the PNAS study, he said.

Barbas said his group has not encountered any significant difficulties in synthesizing programming agents. "Usually there is a site on a therapeutic peptide or small molecule that can be used for linkage" to the diketone, he said.

\section{Ab extensions}

There was less consensus about whether the technology could extend to infectious diseases.

Rickert said the technology's effectiveness against infection needs to be demonstrated, "but it is a perfectly valid extrapolation to make from these findings. The challenge with targeting pathogens will be that they 
are constantly evolving and mutating."

Thus, he said a programming agent should direct antibodies against a conserved site on a pathogen. Otherwise, the agent would be useless once the pathogen mutates-for example, as seasonal flu does.

MediGene's Guenzi was more cautious. "There is no evidence in the paper that this system could be used for non-self-antigens," he said.

Crucell's Uytdehaag agreed that preventing infection with programmed antibodies was a long way off. "For vaccination against viral diseases, it remains to be demonstrated whether programmed antibodies can neutralize-not just bind-a virus," he said.

Uytdehaag said that success in treating malaria, HIV and flu would depend on developing chemical programming agents with high affinity for the pathogens. He agreed with Rickert that such agents should target highly conserved epitopes to prevent escape mutations.

Uytdehaag also said there would be logistical issues in using the programmable antibodies for many infectious diseases.

"The broad and universal applicability of the technology to create instant immunity in the event of, for example, a flu pandemic, would require a pre-existing immunity to the [diketone] in the general population," he said. "Mass immunization early in life to generate a long-lived memory B cell response to the diketone is theoretically possible, but there may be ethical and regulatory issues involved with such an approach."

Rickert disagreed, noting that the diketone used by the Barbas team was inert, did not accumulate in the body and had no known human toxicity.

Lerner concurred with Rickert, adding that the compounds used by CovX and Pfizer to induce the antibody scaffolds have long-standing safety records in humans. Thus, he didn't anticipate significant ethical or regulatory hurdles to generating pre-existing, programmable immunity in the general population.

Barbas said his group has developed a variety of different antibodies that are also suitable for chemical programming and just as versatile as the anti-diketone antibodies. He added that his group is working to advance the technology described in PNAS to treat HIV, influenza and cancer.

Lerner said Scripps holds all of the IP related to chemically programmed antibodies and Pfizer has licensed the rights.

Haas, M.J. SciBX 2(10); doi:10.1038/scibx.2009.388

Published online March 12, 2009

\section{REFERENCES}

1. Wagner, J. et al. Science 270, 1797-1800 (1995)

2. Popkov, M. et al. Proc. Natl. Acad. Sci. USA; published online March 2, 2009; doi:10.1073/pnas.0900147106

Contact: Carlos F. Barbas III, The Scripps Research Institute, La Jolla, Calif.

e-mail: carlos@scripps.edu

COMPANIES AND INSTITUTIONS MENTIONED

Burnham Institute for Medical Research, La Jolla, Calif.

Crucell N.V. (Euronext:CRXL; NASDAQ:CRXL), Leiden, the Netherlands MediGene AG (Xetra:MDG), Martinsried, Germany

Pfizer Inc. (NYSE:PFE), New York, N.Y.

The Scripps Research Institute, La Jolla, Calif. 